\title{
Ventilation characteristics of the built environment and their effects on the urban microclimate
}

\author{
P. A. Fokaides ${ }^{1}$, C. N. Markides $^{2} \&$ M. Neophytou ${ }^{1}$ \\ ${ }^{1}$ Laboratory of Environmental Fluid Mechanics, \\ Department of Civil and Environmental Engineering, \\ University of Cyprus, Lefkosia, Cyprus \\ ${ }^{2}$ Department of Chemical Engineering, Imperial College London, \\ London, UK
}

\begin{abstract}
The thermal conditions within cities, as well as the energy efficiency of buildings, are determined by the urban climate, which is in turn influenced by the thermal-fluid characteristics of the built environment and particularly transfer effects to the atmosphere. A good example of this interaction is the urban heat island phenomenon. In this case heat generated from urban activities has a direct impact on the urban microclimate, resulting in increased night time temperatures and a reduction in the observed temperature range.

In order to understand the interaction between the built environment and the urban microclimate, one needs to consider:

1. The energy efficiency of the built environment and mainly the phenomena of heat transfer through radiation.

2. The ventilation capability of the urban environment and the effects of different building geometries.

The study presented in this paper is concerned with the ventilation of the built environment and involves Particle Image Velocimetry (PIV) measurements under street canyon flow conditions in a flow channel.

Keywords: street canyon, urban heat island, Particle Image Velocimetry (PIV).
\end{abstract}




\section{Introduction - literature review}

Urbanisation and industrialisation have an important influence on urban climates and on the environmental performance of buildings. Increased urban densities result in reduced housing plot sizes, vegetation and trees. In addition, the potential for traffic congestion increases. Air temperatures in densely built urban areas are higher than those of the surrounding countryside. The phenomenon is known as 'heat island' and is especially noticeable in the summer. The urban climate has a considerable impact on the energy consumed in buildings for cooling. Higher air temperatures have a direct influence on building envelope loads due to the increase in conduction loads and radiation loads mainly through fenestration. On the other hand, the potential for utilisation of natural ventilation for occupant health and comfort, as well as for cooling, can be reduced considerably by the higher air temperatures and the lower air velocities at street level. The urban design factors that characterize the climatic aspects of an urban layout are [1]:

- Built density

- $\quad$ Street aspect ratio (height over width)

- Street orientation

- Mineral/vegetal ratio of the non-built area

- Type of vegetation

- $\quad$ Properties of materials, etc.

The term 'street canyon' refers in principle to a relatively narrow street with buildings lined up continuously along both sides. However, the same term has been used to refer to larger streets, also called 'avenue canyons'. In the real world, a broader definition of the term has been applied, including urban streets that are not necessarily flanked by buildings continuously on both sides, allowing thus for some openings on the walls of the canyon. The dimensions of a street canyon are usually expressed by its aspect ratio, which is the height $\mathrm{h}$ of the canyon divided by the width $\mathrm{d}$. A canyon might be called regular, if it has an aspect ratio approximately equal to 1 and no major openings on the walls. An avenue canyon may have an aspect ratio below 0.5 , while a value of 2 would be representative of a deep canyon [2].

A brief literature review of some important investigations of the behaviour of urban flow in street canyons is presented. Dezsö-Weidinger et al. [3] utilised a technique, based on simultaneous PIV and Particle Tracking Velocimetry (PTV), to capture instantaneously both the velocity and concentration fields. The PIV/PVT images were then used to measure the turbulent mass flux vector $\hat{u} / \widehat{c}$ which is determined as the ratio between the mean flow velocity and the mean concentration of a tracer in the flow, and employed to check the correctness of the model widely used for the turbulent mass flux in RANS simulations of street canyon type flows. The measurements in this work verify that the mean concentration shows accumulation in the upstream corner and a stable vortex in the canyon. Concluding on the turbulent mass flux in the street canyon, one can tell that the suggested model seems to be valid in the $2 \mathrm{D}$ shear region but not 
inside the canyon, where the gradient in mean concentration is not aligned with the gradient in mean velocity, where not turbulent diffusion, but the advection is governing.

Caton et al. [4] investigated experimentally and analytically the dispersion mechanisms of a passive tracer in a two-dimensional model of a street canyon. Their principal concern was the concentration transfer between the street and the external flow. The mass fluxes were not only inferred from mean concentration measurements, but also measured directly with PTV. Visualization of the evolution of the concentration field showed the role of the shear layer at the top of the street canyon. Analytical transfer and dispersion models were derived, in agreement with the measurements, demonstrating the importance of external turbulence properties on the transfer. The results presented in this article strongly suggest that the transfer in a street canyon does depend on the structure of the incoming turbulence, i.e. on the local stability conditions and on the upwind buildings.

A recent effort by Simoeens et al. [5] based on idealized laboratory experiments related to the flow field kinematics in and around a street canyon embedded in a turbulent boundary layer were carried out with PIV measurements. A canyon was formed by two square rods of cross-section $\mathrm{h}^{2}$ that spanned the wind tunnel. By varying the canyon opening from $1 \mathrm{~h}$ to $10 \mathrm{~h}$ the authors established and investigated three flow regimes: skimming flow, wake flow and isolated flow. For all canyon openings a primary vortex, with negative vorticity and driven by the external flow above it, was observed. As the canyon was widened to $2 \mathrm{~h}$, a secondary vortex with positive vorticity developed in the downstream canyon corner on the windward side of the downstream obstacle. At an opening of $6 \mathrm{~h}$ a tertiary vortex appeared in the upstream canyon corner on the leeward side of the upstream obstacle. These vortices were not stationary; they moved about their average locations. As indicated by the two-dimensional turbulent kinetic energy for the smaller canyon openings up to $3 \mathrm{~h}$, the lower edge of the shear layer, formed by the separated flow past the upstream obstacle, penetrated into the downstream part of the canyon. As the opening was increased further, the perturbed region extended upstream in the canyon, gradually filling most of it. The Reynolds shear stress above the canyon was negative for all openings. The vertical region where the negative stress level was high widened with increasing canyon opening width, and penetrated into the canyon. A small region of positive stress just above the upstream obstacle was observed for all canyon openings.

\section{Infrastructure - methodology}

The model that was examined in this study comprises two flat horizontal surfaces and a cavity located between them, with a height to depth ratio (h/d) equal to 1 (see Figure 1). The cavity was placed on the channel floor, perpendicular to the flow direction, establishing a two-dimensional problem. The specific height to depth ratio $(\mathrm{h} / \mathrm{d}=1)$ was chosen as representative of typical urban geometries in Cyprus. The height (and depth) of the cavity was equal to 
$60 \mathrm{~mm}$. This dimension was chosen such that the cavity was completely contained within the boundary layer on the channel floor. A fully-developed turbulent boundary layer was created by means of a trip wire located upstream in the inlet element of the modular channel (Figure 2). The cavity/measurement section was placed $4 \mathrm{~m}$ from the trip wire.

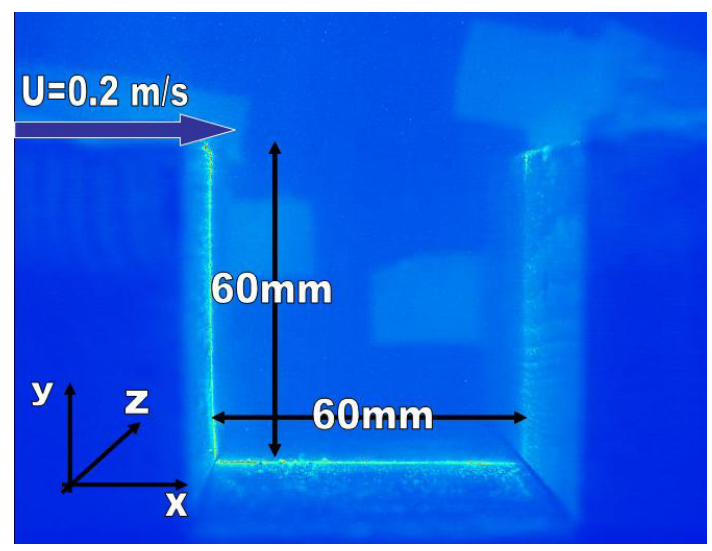

Figure 1: $\quad$ Street canyon model.

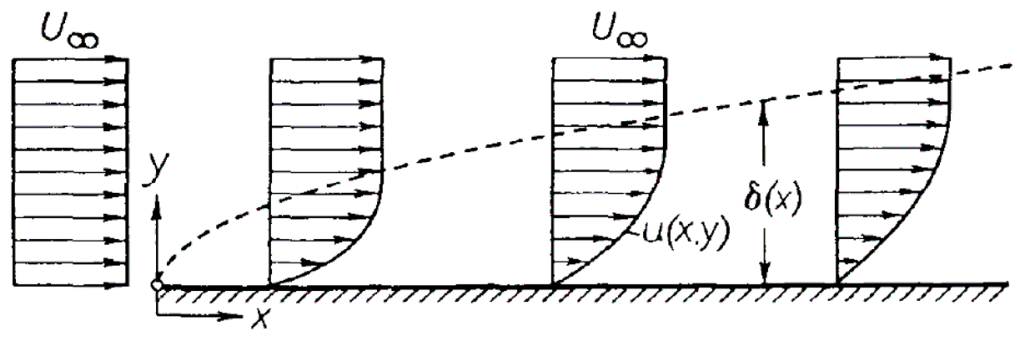

Figure 2: $\quad$ Shear layer in channel flow [6].

The Reynolds number of the boundary layer is determined as:

$$
\operatorname{Re}_{X}=\frac{U_{\infty} x}{v}
$$

where $U_{\infty}$ is the mean free stream velocity, $\mathrm{x}$ is the distance between the onset of the boundary layer and the flow region at which the Reynolds number is determined and $v$ is the kinematic viscosity of the fluid.

In turbulent flows, the thickness of the boundary layer can be estimated by the expression [6]:

$$
\delta=0.14 \times\left[\frac{v}{U_{\infty}}\right] \times\left[\frac{\operatorname{Re}_{X}}{\ln \operatorname{Re}_{X}}\right] \times G\left[\ln \operatorname{Re}_{X}\right]
$$


The thickness of the boundary layer was determined in terms of the following data:

- Flow dimensions: $\mathrm{z}=0.3 \mathrm{~m}, \mathrm{y}=0.35 \mathrm{~m}$

- Free flow velocity: $\mathrm{U}_{\infty}=0.29 \mathrm{~m} / \mathrm{s}$, based on the mean flow and the area of the channel

- Viscosity: $v=1 \times 10^{-6} \mathrm{~m}^{2} / \mathrm{s}$ (water at $20^{\circ} \mathrm{C}$ )

- Distance between measurement point and trip wire: $\mathrm{x}=4 \mathrm{~m}$

The Reynolds number of the free flow in this case is determined based on the building height as well as the mean free stream velocity, and is equal to 17400 . Even though this Reynolds number ensures turbulent pollutant dispersion, it is 1 -2 orders of magnitude smaller than what is required for the recreation of real environmental conditions.

Figure 3 shows the modular flow channel that was employed in this study (Gunt, HM 162 Modular Flow Channel). The channel has a square cross section of $300 \mathrm{~mm} \times 450 \mathrm{~mm}$ and a total length of $7.5 \mathrm{~m}$. However, in the measurements presented in this paper the depth of the water was limited to about $350 \mathrm{~mm}$. The channel has glass side walls, thereby guaranteeing an almost complete view into the measuring section. Further details related to the geometry and the flow field in the modular flow channel are provided in Table 1.

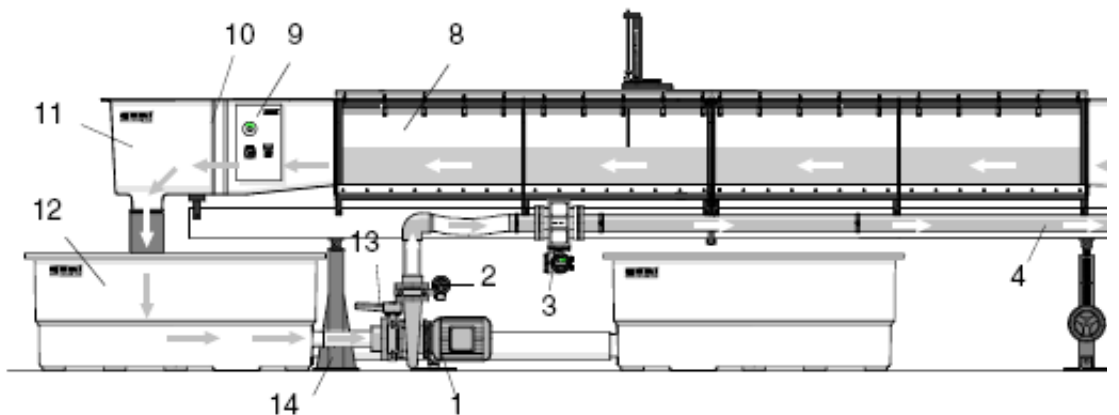

1. Centrifugal pump

2. Shut off valve w. gear system and hand wheel to regulate flow rate

3. Flow rate meter

4. Pressure line
5. Incline adjustment facility

6. Flow rectifier

7. Intake element

8. Modular centre element

9. Switch box
10. Overflow sensor

11. Outflow element

12. Tank

13. Shut off valve

14. Fixed bearing

Figure 3: Gunt, HM 162 modular flow channel.

The principle of the PIV technique is the estimation of the velocity of the tracers added to the flow [7]. The tracer particles are sufficiently small (mean size $9-13 \mu \mathrm{m}$ ) to assume that they follow the flow. To determine the velocity of the tracers, two successive images are recorded. The velocity is determined by evaluating the displacement of the tracers during a specific time interval. The flow is seeded with particles that act as small tracers. A thin laser sheet 
illuminates the part of the flow field, which has to be investigated. The camera placed perpendicularly to the sheet captures two successive images. The PIV technique estimates the displacement of particles grouped in windows, by using cross correlation methods. The technical data of the PIV system that was employed in terms of this work are provided in Table 2.

Table 1: $\quad$ Technical data, modular flow channel, Gunt, HM 162.

\begin{tabular}{|l|c|}
\hline Length & $8.75 \mathrm{~m}$ \\
\hline Range of measuring & $7.50 \mathrm{~m}$ \\
\hline Depth & $300 \mathrm{~mm}$ \\
\hline Height & $450 \mathrm{~mm}$ \\
\hline Water volume & $3.5 \mathrm{~m}^{3}$ \\
\hline Maximum flow rate & $220 \mathrm{~m}^{3} / \mathrm{h}$ \\
\hline
\end{tabular}

Table 2: $\quad$ Technical data of the PIV system.

\begin{tabular}{|c|c|}
\hline \multicolumn{2}{|c|}{ Laser (Litron, Nano L 125-15 - Nd:YAG) } \\
\hline Repetition rate $[\mathrm{Hz}]$ & $0-15$ (per laser) \\
\hline Energy per pulse [mJ] & $125 @ 532$ nm \\
\hline Beam diameter [mm] & 5 \\
\hline Timing jitter [ns] & $<0.5$ \\
\hline \multicolumn{2}{|c|}{ Camera (Imager Pro X 4M) } \\
\hline Sensitivity Range [nm] & $290-1100$ \\
\hline Card size [pixel] & 2048 \\
\hline Pixel size $[\mu \mathrm{m}]$ & 7.4 \\
\hline Time resolution [ns] & 500 \\
\hline \multicolumn{2}{|c|}{ Light sheet optics } \\
\hline Working distance [mm] & 300 \\
\hline Thickness $[\mu \mathrm{m}]$ & 100 \\
\hline Divergence angle $\left[{ }^{\circ}\right]$ & 30 \\
\hline
\end{tabular}

\section{Results - discussion}

Figures 4 to 7 show the results of the PIV measurement campaign. The measurements were performed for a free stream velocity equal to $0.29 \mathrm{~m} / \mathrm{s}$ and all the findings presented in this paper result from the evaluation of 35 consecutive pair of images. The time interval between the consecutive images was offset according to:

- the mean free stream velocity $(0.29 \mathrm{~m} / \mathrm{s})$

- the field of view $(150 \mathrm{~mm} \times 150 \mathrm{~mm})$

- the size of the interrogation window (64 pixels)

and was set equal to $1750 \mu$ s.

Non-dimensional presentation of the results is performed based on the mean free stream flow velocity Uo. Reynolds stresses are normalized by $\mathrm{Uo}^{2}$ and the 
turbulent kinetic energy $\mathrm{k}$ by $1 / 2 \mathrm{Uo}^{2}$. The evaluation of the results was performed by using LaVision's DaVis version 7.2 [8].

Figures 4 and 5 present the measurement of the mean flow velocity vector field:

$$
\bar{U}=\frac{1}{n} \sum_{i=1}^{n} U_{i}
$$

As expected, a major recirculation zone is observed in the street canyon, which is expanded within the whole cavity and reaches the deepest point in the canyon. This recirculation is created by the shear layer between the canopy area on the top of the buildings and the free stream flow, which is interrupted in the region above the street canyon, resulting in the convergence of the flow towards the windward side building.

The direction of the horizontal velocity component at the lower part of the model canyon is opposite to the free stream flow velocity (Figure 4). The core of the major recirculation zone does not coincide with the geometrical centre of the model canyon, but it is found to be slightly displaced towards the windward side building (Figure 4 - detail), consistent with the findings in [3-5].

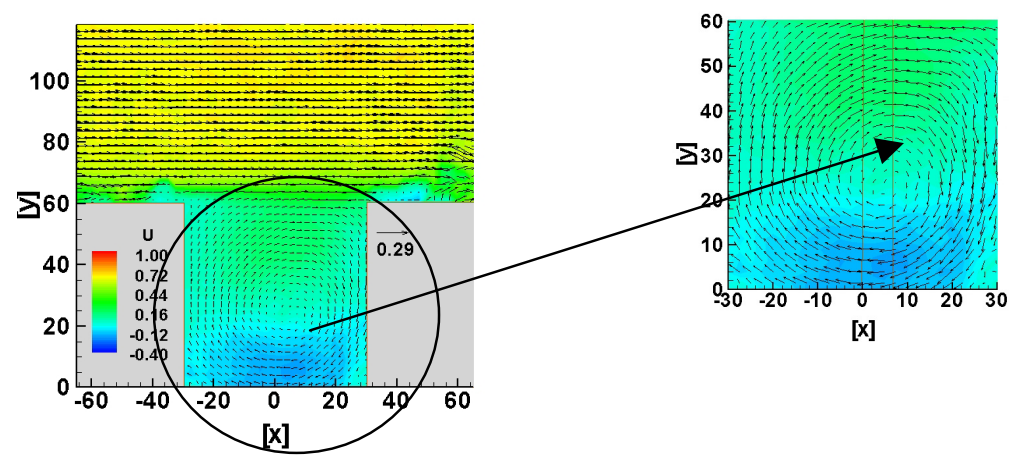

Figure 4: Mean velocity vectors and contour plots of horizontal mean velocity $(\bar{U})$ (left) and recirculation zone detail (right).

The urban canopy is a microscale concept and the specific climatic conditions at a given point are determined by the nature of the immediate surroundings (adjacent streets, buildings, trees etc.). The variables affected are mainly the incident solar radiation, the wind patterns, the long-wave radiation to the sky and the ground temperature, thus isothermal and at a constant flow velocity. In experimental scale, the canopy area conditions are similar to the free stream conditions.

Figure 5 shows the mean vertical flow velocity $(\bar{V})$ in the street canyon. In the shear layer between the flow and the windward side building, the vertical flow velocity is found to be negative, whereas in the leeward side the direction is positive. The core of the major recirculation zone does not coincide with the 
geometrical centre of the model canyon, but it is found to be slightly displaced towards the windward side building (Figure 4 - detail), as was found in [3-5].

From the PIV measurements of the velocity components in the $\mathrm{x}-\mathrm{y}$ plane the spatial distribution of the level of turbulence within and around the canyon is indicated by the contours of the two-dimensional turbulent kinetic energy, nondimensionalized by $\mathrm{U}^{2}$. Figure 6 illustrates its contour for the investigated case. The turbulent kinetic energy per unit mass can be expressed as:

$$
k=\frac{1}{2}\left(U_{R M S}^{2}+V_{R M S}^{2}\right)
$$

where the 'Root Mean Square' is given by:

$$
U_{R M S}=\sqrt{\frac{1}{(n-1)} \sum_{i=1}^{n}\left(U_{i}-\bar{U}\right)^{2}}
$$

In the case of the turbulent kinetic energy, two major zones are observed:

- the first one is extended within the canopy area

- the second one is located in the model canyon

The major difference between these two regions is the magnitude of the turbulent kinetic energy, since the turbulence levels in the canopy area seem to be much higher. The magnitude distribution of the kinetic energy in these two regions reveals the character of the recirculation zone and thus the existence of a stagnation point at the windward side building. This stagnation is expected to act as a pollutant trap in a street canyon, since the pollutant concentration in the canyon is expected to be higher in this region.

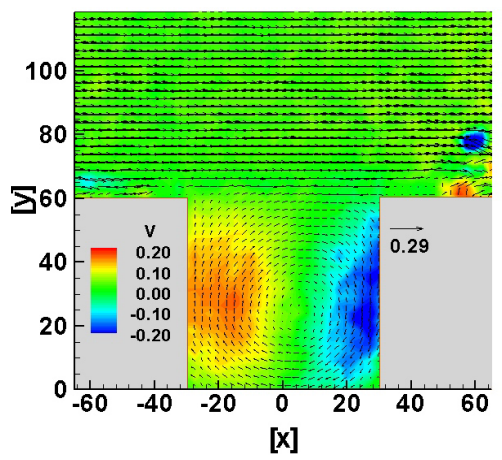

Figure 5: Mean velocity vectors and contour plots of vertical mean velocity $(\bar{V})$.

Another tool in order to evaluate a flow field in the street canyon is the Reynolds shear stress $\overline{\left(U^{\prime} V^{\prime}\right)}$, since it presents the stress tensor in a fluid due to the random turbulent fluctuations in fluid momentum. In terms of the measured data, the Reynolds shear stress is determined by: 

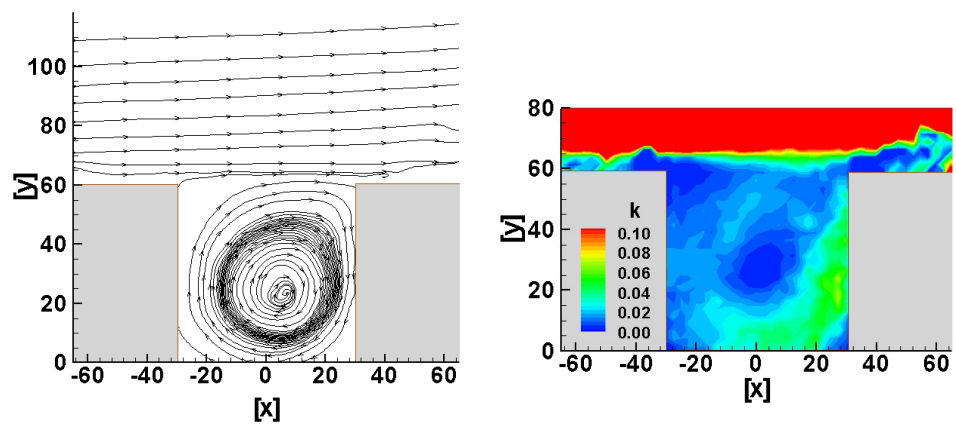

Figure 6: Streamlines of mean velocity field (left) and turbulent kinetic energy contour (right).
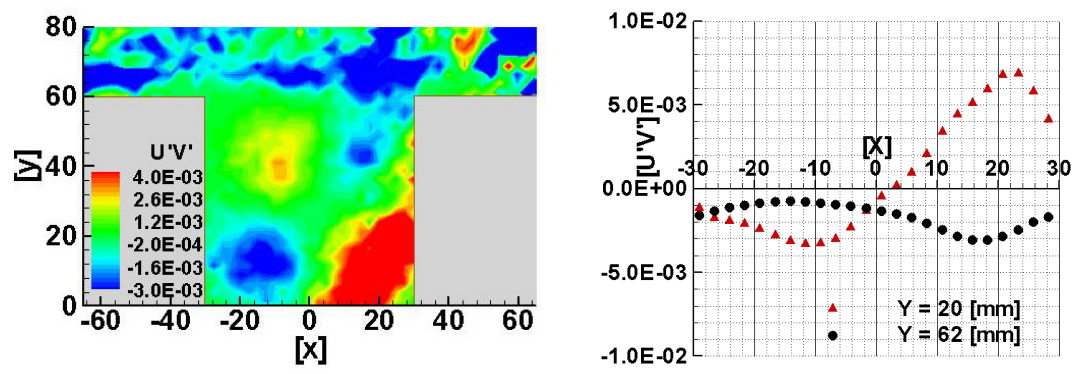

Figure 7: Reynolds shear stresses contour $\overline{\left(U^{\prime} V^{\prime}\right)}$ (left) and Reynolds shear stresses $\overline{\left(U^{\prime} V^{\prime}\right)}$ in the canopy shear layer $(\mathrm{Y}=62[\mathrm{~mm}])$ and in the cavity $(\mathrm{Y}=20[\mathrm{~mm}])$ (right).

$$
\overline{U^{\prime} V^{\prime}}=\frac{1}{n} \sum_{i=1}^{n}\left(U_{i}-\bar{U}\right)\left(V_{i}-\bar{V}\right)
$$

The shear stress for the investigated case is given in Figure 7 . We note that the magnitude of the shear stress in the recirculation core equals zero. This occurs due to the fact that no momentum transport is expected to occur through the recirculation core. The distribution of the shear stresses in the periphery of the recirculation zone is also of interest, and thus the direction of $\overline{\left(U^{\prime} V^{\prime}\right)}$ is in good agreement with the direction of the velocity components. The higher Reynolds stresses appear, as expected, in the area of the maximum turbulent kinetic energy, due to the fact that the turbulent diffusive transport that occurs in this region is maximised. The direction of the shear stresses in the shear layer between the canopy area and the street canyon was found to be negative, and thus towards the canyon. This measurement indicates the tense of the free stream 
to entrain the canyon. However the intensity of this entrainment seems to be low, and thus the ventilation is still to optimize, since the magnitude of the shear stresses within the canyon are up to three times higher than the stresses in the shear layer.

\section{Conclusions}

This study represents a reference work regarding the research progress in the field of the investigation of the interaction between the built environment and the urban microclimate. This study mainly approaches ventilation issues of the built environment by evaluation of PIV measurements under street canyon flow conditions in a flow channel. The measurements were performed for a free stream velocity equal to $0.29 \mathrm{~m} / \mathrm{s}$ and all the findings presented in this paper result from the evaluation of 35 consecutive pair of images. As expected, a major recirculation zone is observed in the street canyon, which is expended within the whole cavity and reaches the deepest point in the canyon. This recirculation is created, mainly due to the shear layer between the canopy area on the top of the buildings and the free stream flow, which is interrupted in the region above the street canyon, resulting to the convergence of the flow towards the windward side building. From the PIV measurements of the velocity components in the $x-y$ plane the spatial distribution of the level of turbulence within and around the canyon was indicated. The magnitude distribution of the kinetic energy in the street canyon reveals the character of the recirculation zone and thus the existence of a stagnation point at the windward side building, which is expected to act as a pollutant trap. Reynolds stresses appear, as expected, in the area of the maximum turbulent kinetic energy, due to the fact that the turbulent diffusive transport that occurs in this region is maximised. The direction of the shear stresses in the shear layer between the canopy area and the street canyon was found to be negative, and thus towards the canyon.

\section{References}

[1] Alvarez, S., et al. (2000) Designing for Summer Comfort, Heat Gain Control and Passive Cooling of Buildings, a European Handbook, European Commission Directorate-General XVII-Energy ALTENER Programme.

[2] Vardoulakis, S., et al. (2003) Modelling air quality in street canyons: a review, Atmospheric Environment, 37, 155-182.

[3] Dezsö-Weidinger, G., et al. (2003) Measurement of the turbulent mass flux with PTV in a street canyon, Journal of Wind Engineering and Industrial Aerodynamics, 91, 1117-1131.

[4] Caton, F., et al. (2003) Dispersion mechanisms in a street canyon, Atmospheric Environment, 37, 693-702.

[5] Simoeens, S., et al. (2007) The flow across a street canyon of variable width-Part 1: Kinematic description, Atmospheric Environment, 41, 90029017. 
[6] Schlichting, H., et al. (2000) Grenzschicht Theorie, Springer Verlag, ISBN3-540-55744-x.

[7] Raffel, M. et al. (2000) Particle Image Velocimetry, A practical guide, Springer Verlag, ISBN-3-540-63683-8

[8] DaVis 7.2 Software, Item-Number(s) Q 1005xxx, Product Manual, La Vision, 2008.

[9] Kakoniti, A., (2008) The effect of radiation heat transfer in the urban heat island phenomenon, using computational fluid dynamics simulation, Master of Science Thesis, University of Cyprus. 